prehensile tail" ; they are flattened and furnished with minute hooks. The radial nerves of the arms are strongly developed. The tube-feet, though quite short, are strongly adhesive.

There is another ophiurid with a very small body, but in the smallness is relative as well as absolute; indeed it "can hardly be said to have a disk" at all. This is ophiocanops fugiens, first made known by Koehler and now re-described from better specimens by Dr. Mortensen. The chief features, unique among ophiuroids, are connected with the minute size of the body. The first is the position of the genital glands as a series of small isolated organs along each side of the arm, each opening separately on the side of the arm. The second is the continuation of the stomach as a sort of radiating intestine out in the arms, in the dorsal space, above the genital organs, for about two-thirds the length of the arm; its walls are much folded and it is supported by a horizontal mesentery. Ophiocanops may be related to the Ophiomyxidæ but it is here separated as a new family.

It is, of course, well known that in some of the ophiuroids with branching arms-the Euryalæ-the genital glands pass into the arms, but the arrangement is quite different from that in Ophiocanops. In a paper describing several specimens from the Indo-Pacific, Dr. Mortensen shows that "all the Trichasterids have the gonads extending into the arms to a various degree". They do not branch, however, but lie side by side like a bundle of telegraph cables.

An ophiurid that has given rise to a good deal of discussion is Ophiopteron, which Ludwig described in 1888 as "eine neue wahrscheinlich schwimmende Ophiuridenform". The reason for the supposition was that the arm-spines were united by a fine membrane, so that each group looked like the foot of a duck. Ludwig's suggestion was therefore generally accepted, but Dr. Mortensen, who has repeatedly observed living specimens, has never seen Ophiopteron swimming. Actually it lives concealed in crevices. The arm muscles are not exceptionally developed, and swimming by an up and down motion of the arms seems out of the question. There remains, none-the-less, the possibility that swimming is effected by a waving movement of the spine-groups, and this is indicated by strong muscles and a definite articulation at the base of the spines. Further observations are required.

This, however, is not the whole story. Dr. Mortensen's re-examination of many specimens referred to Ophiopteron shows that they are the young stage of one or other species of Ophiothrix. All species of Ophiothrix do not pass through an Ophiopteron stage, but why any should do so, and what may be the use of the "fins", are questions still to be solved.

\title{
Manufacture of Phosphoric Acid
}

$I^{\mathrm{N}}$ the Chemiker-Zeitung of September 6, Dr. Carl Heinrich discusses at length the merits of the two main processes which are used for the manufacture of phosphoric acid from calcium phosphate. The essential features of these two processes, which may be conveniently termed the wet and dry processes, are set forth in textbooks of inorganic chemistry. In the wet process, the phosphate is decomposed by sulphuric acid, whereas in the dry process, silica replaces sulphuric acid and the furnace may be heated either externally or by means of an electric resistance. In view of the increasing importance of the product as a fertiliser, it is desirable that a more complete comparison of these two processes should be carried out.

One of the chief drawbacks to the wet process is the solubility of calcium sulphate in the liquor. Nevertheless, it is possible to extract 95-98 per cent of the phosphoric acid by modern decantation methods. This acid may still contain calcium sulphate, sulphuric acid and other impurities, but these are without much significance if the acid is to be used for the manufacture of fertilisers. But for many other purposes a much higher degree of purity is necessary, and the dry process must then be adopted. Thus iron and aluminium are difficult to eliminate from the acid solutions, whereas unless they are present in excessive amount they cause no trouble in the dry process.

In the United States, the dry process is generally carried out in one operation and the electric furnace is used. In Germany, on the other hand, there are usually two stages and external heat is applied. In the former method, the vapours of phosphorus and carbon monoxide are burned in the upper part of the furnace, the phosphorus pentoxide being afterwards condensed in a separate chamber. In the twostage process, the phosphorus is not burned until its vapour has been completely separated by condensation from other vapours, and the carbon monoxide is then available as fuel. In both methods, considerable difficulty is caused by the highly corrosive nature of the hot phosphorus pentoxide vapour. The electric furnace gives a much higher concentration of phosphorus and its oxide than the other furnace, but it must be pointed out that a one-stage process is not thermally economical as much heat is necessarily wasted. On the other hand, the electric furnace is not so well adapted to a two-stage process since the yield of carbon monoxide is usually small, so that its heat of combustion cannot be utilised.

Several advantages are claimed for the externally heated furnace and the two-stage process. Thus by eliminating air from the first chamber, a high temperature can be employed without any danger of forming the obnoxious colloidal mist of phosphorus pentoxide vapour, which wili neither dissolve readily nor condense completely without the application of the costly Cottrell high-tension discharge. Moreover, any loss which might arise from the adoption of an additional operation is much more than compensated by the elimination of the loss caused through corrosion of the silicate lining of the furnace by the hot phosphoric oxide. The vapours of phosphorus and carbon monoxide are then completely separated and the former is burned in air while the carbon monoxide is used as fuel.

Whilst a direct comparison of the two methods under comparable conditions is not available at present, the author inclines to the view that the wet process is the more economical, whenever it can be usefully applied, although the product is apt to be less pure. On the other hand, since no steam is involved in the dry process, the latter can be adapted to the production of phosphoric acid of any desired concentration without the use of evaporation plant. 